\title{
Optimization of Wired Genetic Antenna Using Genetic Algorithm
}

\author{
Renu Kumari ${ }^{1}$, Tarak Nath Paul ${ }^{2}$ \\ ${ }^{I}$ (Faculty of Science and Technology Department, ICFAI University, Jharkhand, INDIA) \\ ${ }_{2}^{2}$ (Faculty of Information Technology Department, ICFAI University, Jharkhand, INDIA)
}

\begin{abstract}
The essence of the paper is to optimize wired antenna with the help of Genetic Algorithm. Genetic algorithm helps to design new antennas which are in great demand for wireless applications and also used to optimize different parameters of traditional antennas. The new wired antenna has been designed with the concept of self similar geometry of fractal antenna. A self similar geometry provides configuring the whole antenna figure as copies of a specific part of antenna. Due to this geometry, the resultant structures have elevated the characteristics like small size, space filling and multiband. A new equation for scattering parameters in terms of current and voltage has been analyzed with the help of Matlab. The results of the proposed work have satisfactory outcomes in the frequency range from $2.4 \mathrm{GHz}$ to $3.65 \mathrm{GHz}$.
\end{abstract}

Keywords: Wire Antenna, Fractal antenna, Scattering Parameter and Genetic Algorithm.

\section{Introduction}

Antennas are most essential and significant elements of any wireless communication system. Antennas available with greater bandwidth and smaller dimension are required by modern telecommunication system. Research on customized antennas is on full swing with extended improvement on its characteristics. Traditional design and optimization of antenna is more complex, time taking and laborious process using analysis and simulation. Genetic Algorithm (GA) is very helpful tool for the designer to design the required antennas at fewer easy steps [1]. The integral ability of GA is to optimize formal design as well as to reproduce of its own. This makes GA an ideal tool to design an automated wired antenna for various and difficult application. Therefore GA is an appropriate method to design requisite antenna with few iterations.

This paper draws attention towards genetic algorithm for optimization of genetic antenna with wire. In this work an impressive method is implemented to calculate the scattering parameter of wired genetic antenna. The model includes description for the reproduction of a new type of wired antenna by chromosomal recombination of two different types of antennas. The expression for scattering parameters of newly designed antenna is derived by the help of Matlab. Therefore a compact description of the wired genetic antenna in terms of the set of S-parameters is discussed.

The outline of paper is as follows, the basics of Antenna are described in section 2. In section 3 focuses on the GA, describes the different procedures followed in genetic algorithm with flow chart diagram. In section 4 and 5, concentrated on the optimization of wired antenna by the help of Genetic Algorithm. Here chromosomal representation of antenna design has been discussed. Section 6 presents the new equation to calculate the scattering parameter of newly designed antenna. Section 7 presents the results and calculations of scattering parameters of wired antennas by the help of Matlab. And finally conclusion of the research and the future work is discussed in Section 8.

\section{Antenna Basics}

The function of an antenna is to convert a Radio Frequency Signal to a propagating electromagnetic wave coming from a transmitter or convert a propagating wave to Radio Frequency Signal in a receiver [2]. There are different types of antennas and each of them has different structures which express different parameters and parameters of electromagnetic wave fonts. The focus of this paper is on wired genetic antennas. Any good conductor antenna whose length is longer than its width is termed as wire antenna.

\subsection{Basic Properties of Antennas}

A large flat piece of metal is used below the wired antenna to reflect the radiation coming from it, which is known as ground plane. Due to the ground plane, the gain and directivity of antenna has been increased because it works like a reflector for the antenna. It can also decrease the required height and dimension of the wired antenna. The wired antennas are designed to mount on the top of any plane surface than base of that surface act as a ground plane.

Scattering parameters are very important as it is easy to work with it and measurement of it can be performed easily at high frequencies. S-parameter is a kind of power wave definition that discusses input-output parameters of the network in the sense of incident and reflected waves [3]. 

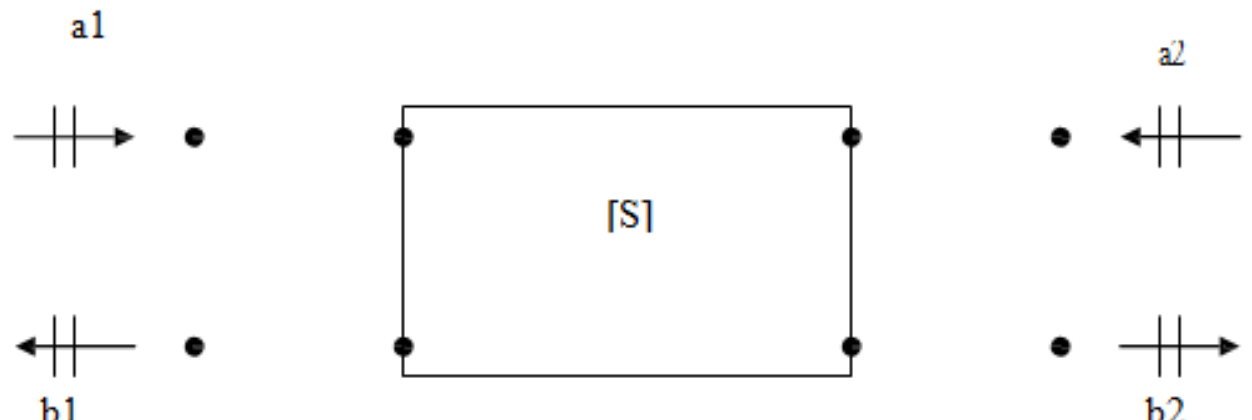

Figure - 1: Scattering Parameters with Sign Convention

According to sign convention shown in figure -1, the $\mathrm{S}$ parameter in matrix form can be written as,

$S=\left|\begin{array}{ll}S_{11} & S_{12} \\ S_{21} & S_{22}\end{array}\right| \quad$ (ScatteringMatrix)

The incident waves 'a', reflected waves ' $b$ ' and $\mathrm{S}$ parameters can be related as follows

$\left|\begin{array}{l}b_{1} \\ b_{2}\end{array}\right|=\left|\begin{array}{ll}S_{11} & S_{12} \\ S_{21} & S_{22}\end{array}\right|\left|\begin{array}{l}a_{1} \\ a_{2}\end{array}\right|$

There will be no reflected waves from the antenna, when load impedance is matched with the input impedance i.e, $\mathrm{Z}_{\mathrm{L}}=\mathrm{Z}_{0}$. This condition provides the actual measurements of the $\mathrm{S}$-parameters

$S_{11}=\left.\frac{b_{1}}{a_{1}}\right|_{Z_{L}=Z_{0}}=$ reflection coefficient

$S_{21}=\left.\frac{b_{2}}{a_{1}}\right|_{Z_{L}=Z_{0}}=$ transmission coefficient

The transfer of power between the transmission lines and the antenna is measure with the help of input impedance. The transfer occurs when both the input impedances of antenna and the transmission line tallies. If it doesn't tallies, the reflected waves will transmit back to the energy source which is generated by the antenna itself. The discussed reflection process reduces the efficiency of the entire system of the antenna.

The ratio between the maximum and the minimum voltage along transmission link is known as Voltage Standing Wave Ration (VSWR). VSWR can be evaluated from the level of reflected upon incident waves. The efficiency of antenna depends on how closely the input impedance tallies with the transmission line. Increase of VSWR implies the increase of the mismatch in between transmission line and antenna, vice - versa.

\section{Genetic Algorithm (Ga)}

Genetic Algorithm is one of the best functional evolutionary computation methods has been used to optimize the performance of the required antenna. It helps in creating new type of antenna. Evolutionary Computing was bought into lime light by I. Rechenberg in the year of 1960 [4]. Genetic Algorithm is invented by John Holland with his students at University of Michigan in the year of 1975. Genetic Algorithm is a heuristic search algorithm or an optimization technique which consider a mating pool and from that pool individuals are selected and mutated to achieve the new generation. Individuals from the new generation will become the best solution for the problem.

Genetic Algorithms work on search algorithms using the mechanism of natural selection. It follow the theory of "Survivals for the fittest" given by Charles Darwins. The best individuals will become the randomized information interchange. The elite individuals will replace the existing population in the pool.

The algorithm commence with a large number of randomly generated chromosomes in the pool. For genetic Algorithm, chromosomes are used as the basic building blocks. A chromosome is a binary representation of the individual and they will be treated as parents. Most fitted chromosomes are allowed for mating. By using the genetic algorithm operator named crossover the selected parents reproduces. In this phenomenon crossover is performed at any point. When new individuals are produced then the process of crossover terminates. Mutation plays a next vital role in Genetic operation. It is a random alteration in the chromosome. Then the fitness of the chromosome is evaluated. The fitted chromosomes are accepted and unfitted chromosomes are discarded. This phenomenon continues until a satisfied solution is reached. The following flowchart will represent the procedure of the genetic algorithm shown in figure -2 . 


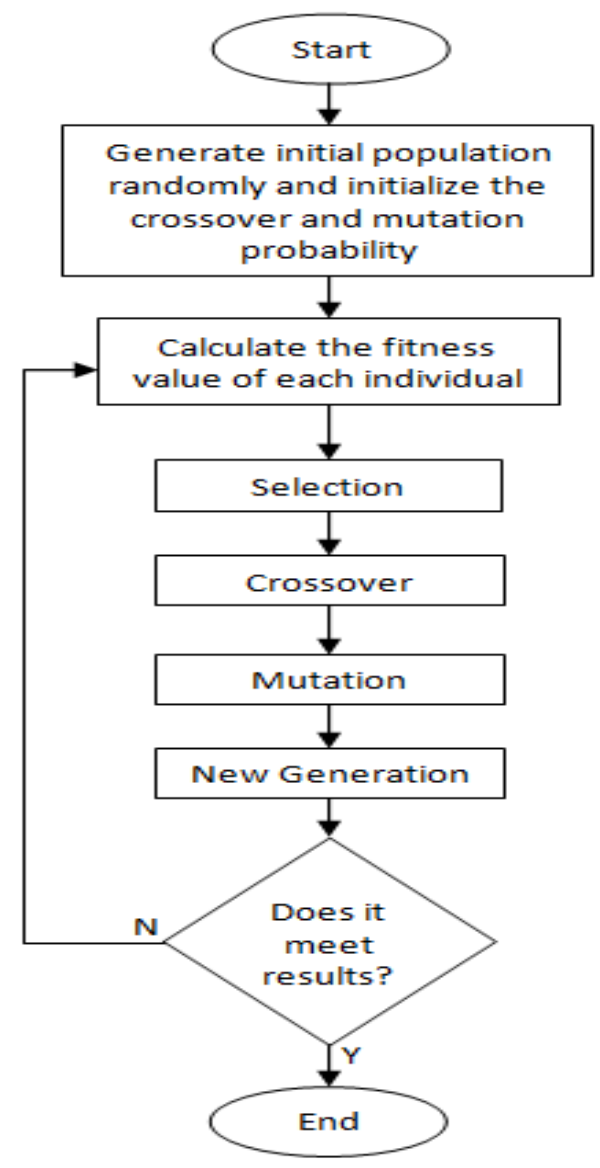

Figure - 2: Flow Chart of Genetic Algorithm Optimization [5]

\section{Antenna Configuration By Genetic Algorithm Procedure}

This paper describes optimization of newly designed wire antenna by the help of genetic algorithm. Mostly antenna optimization starts with basic parameters of conventional antennas. Genetic algorithm provides numerous solutions to create new antenna that is best fitted to required design. To design the required antenna, two different antennas wire antenna and fractal antenna are selected as parent chromosomes.

4.1 Basic Steps of Genetic Algorithm for Optimization of New Antenna

Initialization: Initially to form a population, different individual solutions are randomly selected from the pool of chromosomes. The different properties of wired antennas are used to create the initial population are given below:

A: Impedance - It is measure of voltage to the current ratio at the input terminal of antenna [6].

B: Frequency - Frequency is measure of how fast a wave is oscillating in an antenna.

C: Ground Plane - The antenna is mounted over a large piece of metal that helps to increase the gain of the antenna is known as ground plane.

D: Radiation Pattern - It defines distribution of radiated power emitted by antenna. And also gives idea about which direction the radiation is maximum.

E: Material - Different materials are used to construct the antenna. Mostly glass fiber, woven glass, ceramic etc are used to design the antenna.

F: Polarization -If the electric field of an electromagnetic wave has made directional then the wave is said to be polarized. Omni directional antennas always have vertical polarization.

G: Radiation Efficiency - It describes the relation between the radiated power and the incident power of the antenna. An antenna has said to be less efficient if most of the power has reflected away.

H: Gain - It describes how efficiently an antenna transforms the available power at its input terminal to the power radiated in a given direction.

I: Bandwidth - The operational frequency range of antenna is known as bandwidth. It is difference between the minimum and maximum frequency of antenna.

J: Field Region - The three effective field regions of antennas are - Reactive near field, Radiating near field and Far field. Out of these three field region, Far field region is most important because it defines antenna's radiation pattern. 
K: Side lobes - The plot of energy of antenna radiation in a direction other than the preferred direction is known as side lobes.

The different properties of Fractal antennas are used as an initial population are given below:

p: Scattering Parameter - It express the input and output relation of a network with respect to incident and reflected power waves.

q: Beam width - Beam width of antenna is usually mean half power beam width. It is measure of decrease in radiation pattern of the antenna from the maximum value of a main beam [6].

r: Return loss -The return loss of antenna is described by the logarithmic ratio of the reflected power to the incident power of antenna.

s: Effective Aperture - It defines the amount of power is occupied by effective area of antenna.

t:Length - Length of antenna is always taken as $1 / 2$ or $1 / 4$ of the wavelength of radiation waves.

$\mathrm{v}$ : Temperature - It is a parameter that depends on the thermal environment in which antenna is placed.

w: Current (I) - Current flows through the antenna is minimum at the end point and maximum at the feed point.

$\mathrm{x}$ : Voltage $(\mathrm{V})-\mathrm{It}$ is maximum at the feed point and minimum at the end point of antenna.

y: Location of antenna - It describes the height of the antenna from the ground/sea level.

z: Directivity - It describes directionality of radiation pattern of antennas. An antenna is said to be have zero directivity if it radiates equally in all directions.

Considering the above properties a group of chromosomes are constructed for the mating pool as shown in figure-3. From where two individuals will be considered for cross over.

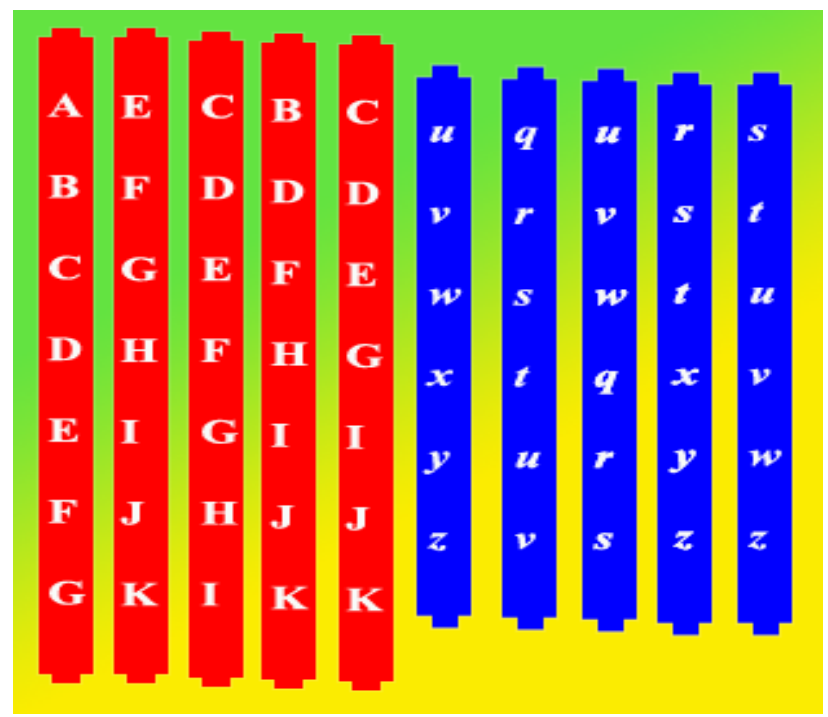

Figure - 3: Pool of Chromosomes

Selection: During every consequent genesis, a part of the existing population is considered for breeding of the next generation. Individual chromosomes are considered through a fitness-function, where filtered individuals are expected to be selected.
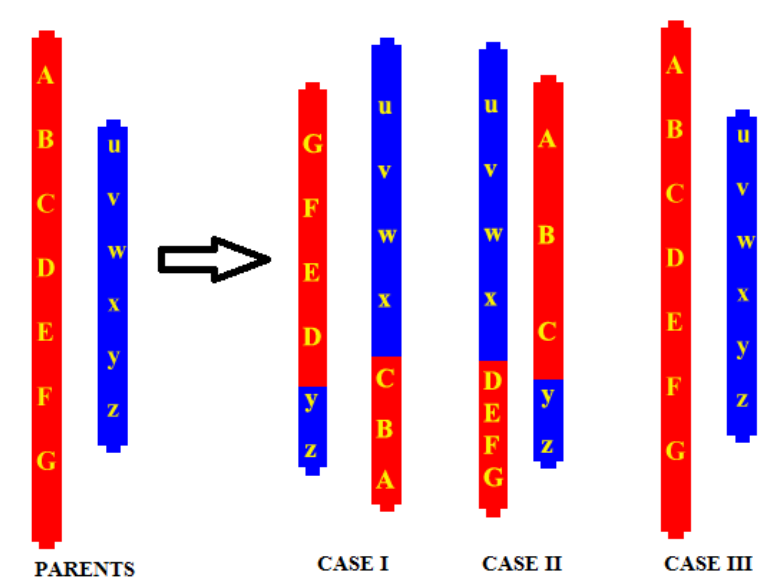

Figure - 4: Recombination of Chromosomes 
Reproduction: In this step second generation population of solutions are considered from the selected pool through genetic operators like crossover (recombination), and/or mutation. Due to the genetic recombination of initial solutions of wired and fractal antenna a new generation with three different cases is produced. These three newly generated cases are given below [4].

Case I - In this case, children chromosomes are different than parent chromosomes. One is generated by the combination of chromosomes [G-F-E-D-y-z] and [u-v-w-x-C-B-A]. Out of these two, the second combination $[\mathrm{u}-\mathrm{V}-\mathrm{w}-\mathrm{X}-\mathrm{C}-\mathrm{B}-\mathrm{A}]$ gives the best fitted solution because it combines the basic necessary parameters of the two parent antennas. With the help of this new combination, an antenna can be designed having reduced size and wider bandwidth.

Case II - In this case, the two children chromosomes [u-v-w-X-D-E-F-G] and [A-B-C-y-z] do not possess the qualified quantities to become fitted individuals. This will provide improper outcomes, so these chromosomes cannot be considered as next generation's chromosomes.

Case III - As the next generations of chromosomes are identical to the parents, so their performance will be similar to parents. Eligibility to be in the pool of next generation for these chromosomes is violated, these chromosomes are discarded.

Termination: The best fitted solution for configuration of new antenna has been found in case I because it has all the properties that required for new antenna. Others solution does not satisfy the minimum criteria so that they have been terminated.

\section{Antenna Design}

A new antenna has been designed with the help of the child chromosome [u-v-w-X-C-B-A] produced by case I. A new monopole wire antenna with four identical arms is used here to achieve the basic design requirement. To design this wired antenna genetic algorithm develops few properties of single arm and configure the complete antenna using four identical copies of the each arm as shown in figure - 5 .

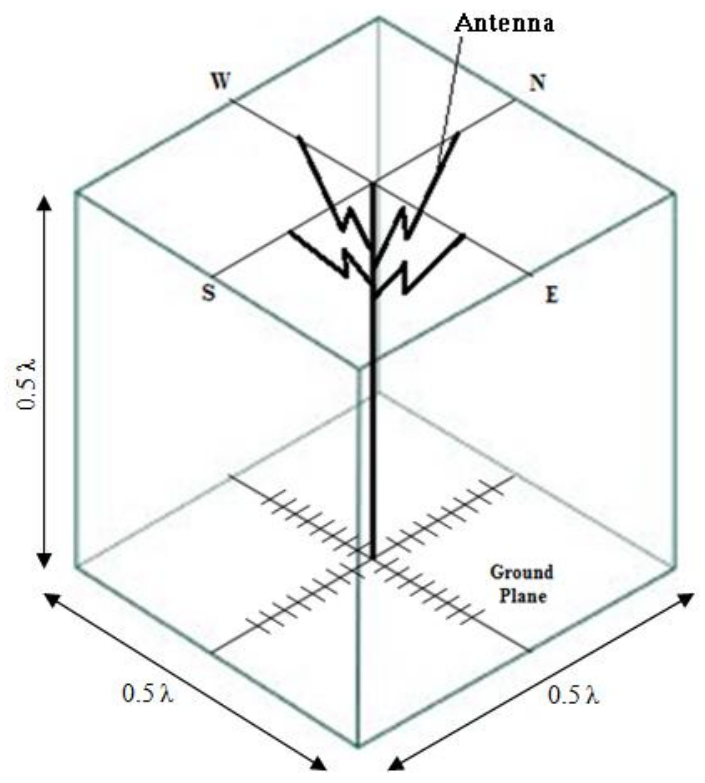

Figure - 5: Optimized Wired Antenna

The antenna is designed by initially using a feed wire at the centre and connecting side arm wires. The base of feed wire is mounted at the centre of the ground plane. To design a four way symmetric antenna, all four arms of wired antenna are of same size and length. This can be achieved by generating other arms of antenna by self similar geometry of one arm and each arm are placed in a quadrant through a rotation of $90^{\circ} / 180^{\circ} / 270^{\circ}$ apart [7]. This technique of self similar geometry of fractal antenna is very useful for the size reduction of the antenna and also enhances the bandwidth of it.

\section{S-Parameters Of Designed Antenna}

Scattering parameter is directly proportional to the amount of current ' $\mathrm{I}$ ' and voltage ' $\mathrm{V}$ ' flowing through the antenna as shown in figure -6 . 


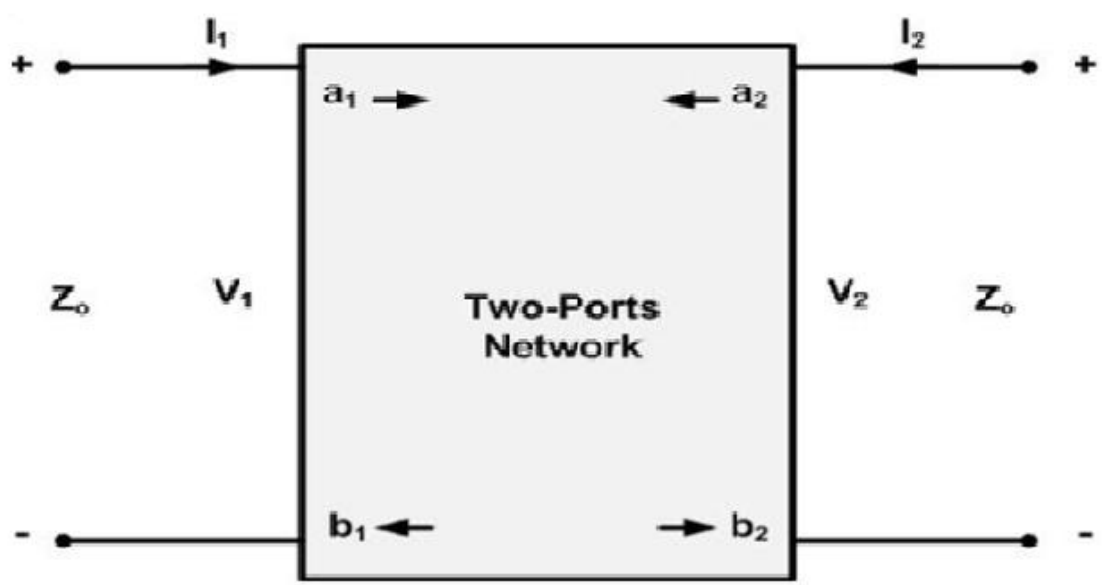

Figure - 6: Scattering Parameter in Terms of Current and Voltage

Therefore, $\mathrm{S}$ - parameter can be expressed as

$$
\begin{aligned}
& {\left[\begin{array}{ll}
S_{11} & S_{12} \\
S_{21} & S_{22}
\end{array}\right] \propto\left[\begin{array}{l}
I_{1} \\
I_{2}
\end{array}\right]\left[\begin{array}{l}
V_{1} \\
V_{2}
\end{array}\right]} \\
& {\left[\begin{array}{l}
S_{11} \\
S_{22}
\end{array}\right]=\operatorname{Re} \text { flection } \text { co }- \text { efficient }=c} \\
& {\left[\begin{array}{l}
S_{21} \\
S_{12}
\end{array}\right]=\text { Transmission co }- \text { efficient }=t}
\end{aligned}
$$

$\mathrm{S}_{11}, \mathrm{~S}_{21}, \mathrm{~S}_{12}, \mathrm{~S}_{22}$ are the coupling coefficient between the transmitting and receiving antenna. Scattering parameters also depends on the temperature ' $T$ ', because according to Wein's law wavelength is inversely proportional to the temperature

i.e $\quad \lambda_{\max }=\frac{b}{T} \quad$ or $\quad \frac{1}{f}=\frac{b}{T}$

Here ' $\mathrm{b}$ ' is constant of proportionality and is equal to $2900 \mu \mathrm{m} . \mathrm{K}$.

Therefore, the fitness function equation of antenna optimization is:

$$
\left[\begin{array}{l}
S_{11} \\
S_{21}
\end{array}\right]\left[\begin{array}{l}
S_{12} \\
S_{22}
\end{array}\right]=\frac{b}{T}\left[\begin{array}{l}
I_{1} \\
I_{2}
\end{array}\right]\left[\begin{array}{l}
V_{1} \\
V_{2}
\end{array}\right]
$$

According to relation of reflection and transmission co-efficient, if a wave of unit amplitude is incident onto the boundary, then relation between transmitted ' $t$ ' and reflected ' $c$ ' wave is given by

$\mathrm{t}=1+\mathrm{c}$

Therefore,

$\left[\begin{array}{l}S_{21} \\ S_{12}\end{array}\right]=1+\left[\begin{array}{l}S_{11} \\ S_{22}\end{array}\right]$

Now, after substituting the value of equation (4), equation (6) becomes

$\left[\begin{array}{l}S_{21} \\ S_{12}\end{array}\right]=1+\frac{b}{T}\left[\begin{array}{l}I_{1} \\ I_{2}\end{array}\right]\left[\begin{array}{l}V_{1} \\ V_{2}\end{array}\right]$

Therefore, from the above equation the value of reflection co-efficient is

$$
\begin{aligned}
& {\left[S_{11}\right]=\frac{b}{T}\left[I_{1} V_{1}+I_{1} V_{2}\right]} \\
& \text { or }\left[S_{11}\right]=\frac{1}{f}\left[I_{1} V_{1}+I_{1} V_{2}\right]
\end{aligned}
$$


$\left[S_{22}\right]=\frac{b}{T}\left[I_{2} V_{1}+I_{2} V_{2}\right]$

or $\quad\left[S_{22}\right]=\frac{1}{f}\left[I_{2} V_{1}+I_{2} V_{2}\right]$

And value of transmission co-efficient is

$$
\begin{aligned}
& {\left[S_{21}\right]=1+\frac{b}{T}\left[I_{1} V_{1}+I_{1} V_{2}\right]} \\
& {\left[S_{12}\right]=1+\frac{b}{T}\left[I_{2} V_{1}+I_{2} V_{2}\right]}
\end{aligned}
$$

\section{Results And Discussions}

The designed wired antenna has been simulated using Matlab. All of the scattering parameters presented in this paper are referenced to $\mathrm{Z}_{0}=50 \Omega$. The antenna is suspended over an infinite ground plane at a height of $\mathrm{h}=0.50 \mathrm{~cm}$ and length $\mathrm{l}=0.30 \mathrm{~cm}$. The simulated $\mathrm{S}$-parameter is plotted against frequency, current and temperature as shown in below Figure - 7. The designed antenna shows good results on frequency range from $2.4 \mathrm{GHz}$ to $3.6 \mathrm{GHz}$. The new antenna structure has $40 \%$ bandwidth. It shown from graph that the return loss is very less therefore it supports the multiband applications.

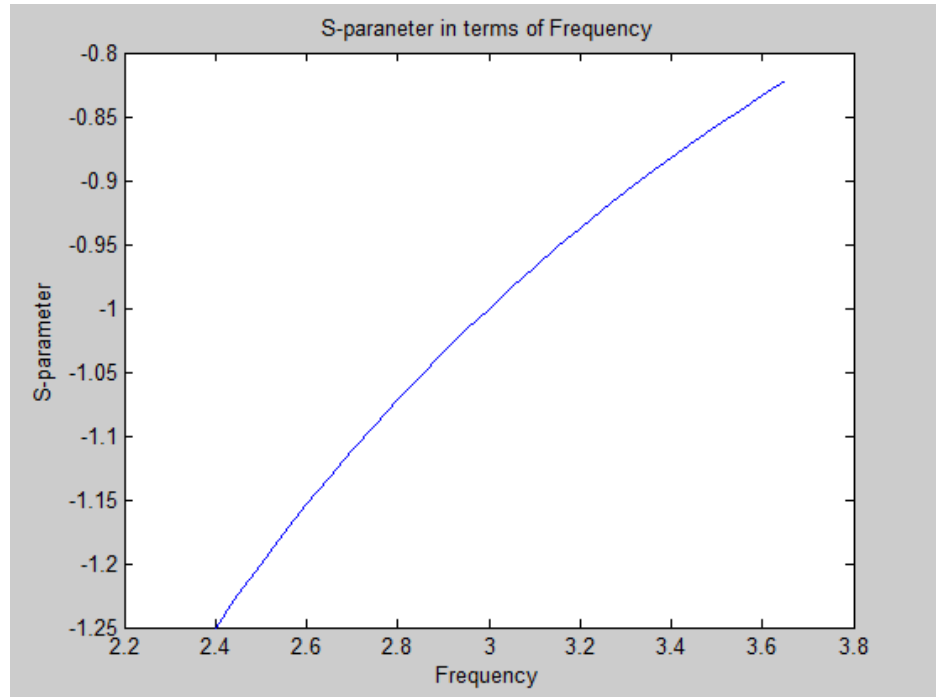

Figure - 7(a): Frequency vs $S_{11}$

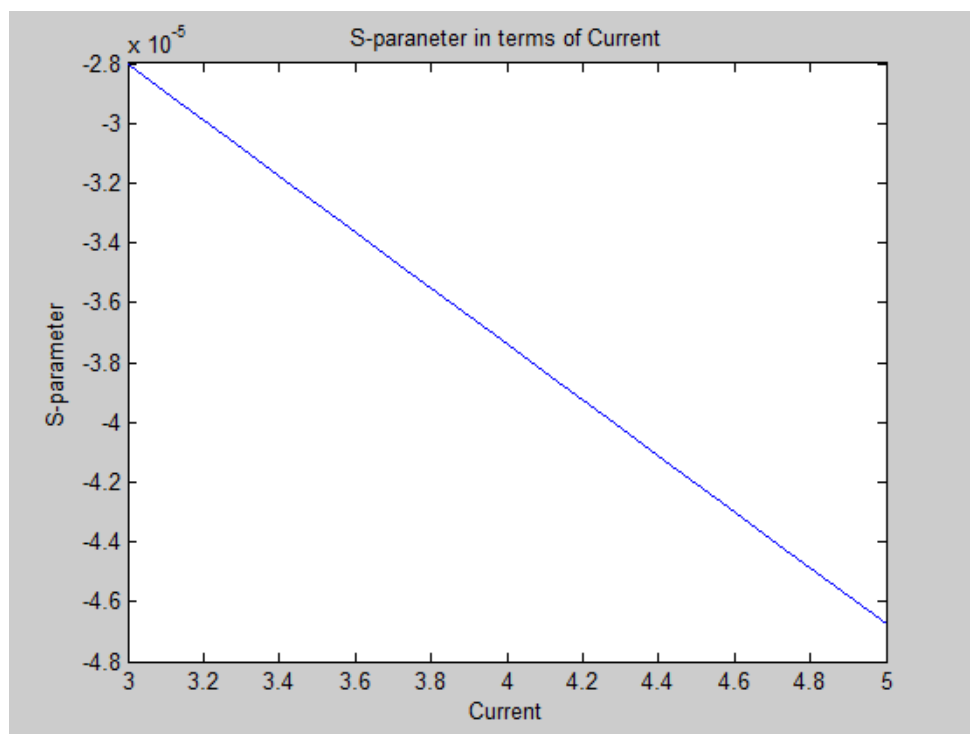

Figure - 7(b): Current vs $S_{11}$ 


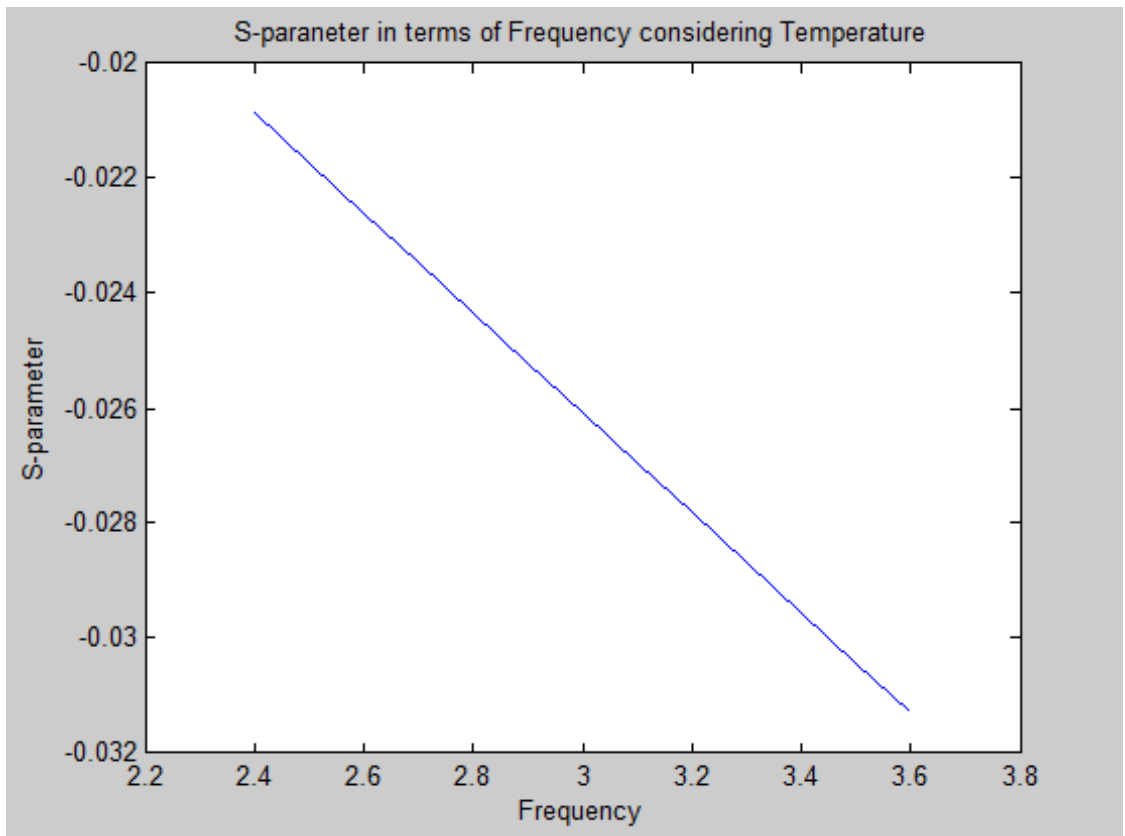

Figure - 7(c): Frequency vs $S_{11}$ using temperature equation

\section{Conclusion And Future Work}

By application of GA, a new wired genetic antenna has been designed. Further description of antenna has been given in terms of scattering parameter. As can be analyzed from the results, antenna shows good characteristics to work for many wireless applications. Because it's offering a low return loss and $40 \%$ band width. As antenna is designed with the concept of self similarity geometry, so it supports the small size and multiband requirements.

As other parameters of antenna has not discussed here. Therefore, description of antenna parameters in terms of $\mathrm{s}$ - parameter network is an extension of this research work.

\section{References}

[1]. Linden D. S., Antenna Design Using Genetic Algorithms, Ph. D Thesis, Ashburn VA, AFIT, 1997.

[2]. Kumari Renu, K. Mamta, Singh R. K., Gain \& Bandwidth Enhancement Of Antenna Using Numerical Experimentation, International journal of research science and management, 3(2), 2016, 56-60.

[3]. Kumari Renu, K. Mamta, Network Analysis Of Antenna Based On Scattering Parameters, International Journal of Industrial Electronics and Electrical Engineering, 3(2), 2015, 1-5.

[4]. Paul, T. N., Studies on Shortest Path Using Genetic Algorithm, Ph. D Thesis, University of Burdwan, West Bengal, INDIA, 2014.

[5]. Saxena, N. K., Khan, A., Pourush, P. K. S, Kumar, N., Application of genetic algorithm for optimization of important parameters of magnetically biased microstrip circular patch Antenn, International Journal of Software Engineering and Applications, vol. 4, 2011,129-13.,

[6]. Balanis, C. A., Antenna Theory: Analysis and Design. (NewYork, Wiley, 1997).

[7]. Linden, D.S., Wire Antennas Optimized in the Presence of Satellite Structures using Genetic Algorithms, Proceedings of the IEEE Aerospace Conference, Big Sky, MT, 18-25 March 2000.

[8]. Weile, D. S., Michielssen, E., Genetic algorithm optimization applied to electromagnetic - A review, IEEE Trans. Antennas Propag, E. vol. 45, no. 3, pp. 343-353, March 1997.

[9]. Gately, A. C., Stock, Jr., D. J. R., Cheo, B. R., A network description for antenna problems, Proc. IEEE, 56n7, pp. 1181-1193, 1968. 